\title{
Numerical Study of Frontal Collision Effects on an Occupant's Safety, in Autonomous Vehicles, with Non-Standard Seating Configurations
}

\author{
Abbas Talimian, Jan Vychytil \\ Biomechanical human body models, New Technologies Research Centre, \\ University of West Bohemia, Univerzitní 2732/8, 30100 Plzeň, Czech Republic, \\ talimian@ntc.zcu.cz,jvychyti@ntc.zcu.cz
}

Abstract: For a long journey, the occupants of highly autonomous vehicles (HAV), with non-standard seating, can communicate easier. The question is... Are non-standard seating configurations as safe as standard seating in a frontal crash? The Virthuman model is used to answer this question. It is a multi-body system (MBS) model that helps run simulations faster, however, it lacks some outputs. A $30 \mathrm{~km} / \mathrm{h}$ frontal acceleration pulse is applied to $H A V$ 's interior and a program determines injury indexes for Virthuman's body parts, then returns to simulation' settings, without considering injuries of the bodies' internal organs. Living Room seating provides safer conditions for its occupants in a frontal crash.

Keywords: fully automated vehicle; Virthuman model; non-standard seating configurations; sledge test simulation; injury index assessment

\section{Introduction}

Currently, a vehicle's occupants can move seats forward or backwards for adjustment. Another possible option for them - usually for occupants in the front row - is leaning a seat's back for relaxation. All of the occupants' sit facing the front windshield and we call this the "standard" seating configuration. But highly autonomous vehicles (HAV) use automated driving systems (ADS) and so far, they do not need drivers for controlling cars manually. In the absence of the emergency brake and gearbox handles and with no steering wheel, occupants can rotate their seats easily to get more legroom and have more freedom, to either communicate with other passengers, or perform various activities. A combination of occupants' seating positions in a highly autonomous vehicle, makes for nonstandard seating configurations.

An interview that gauged an occupants' interest for long travel scenarios in a vehicle, found that almost $80 \%$ of participants prefer to be in a non-standard 
seating configuration [8], while $42 \%$ of Chinese passengers preferred to be in nonstandard seating configurations, from a survey that investigated their seating preference in HAVs [13]. Autonomous vehicle's non-standard seating configurations have different types, but the most desirable ones for occupants are the Face-to-Face and the Living Room configurations. In the Face-to-Face case, the front row passengers' seats are rotated 180 degrees around a vertical axis, perpendicular to the vehicle's floor and face backwards. Meanwhile, the rear row occupants' seats keep their face forward toward the front windshield. If both front and rear row seats are rotated on a vertical axis perpendicular to the vehicle's floor, somehow they are seen like a cross sign, from the top view, which is why we have named it the "Living Room" position.

Rotatable seats could define an active seat rotation strategy to study occupants' safety in automated vehicles. In this concept, the orientation of a passenger's seat is modified during a pre-collision [7] [21]. Seat rotation hypotheses may not always work successfully. For instance, let us suppose an occasion when an autonomous vehicle is driven on a road and suddenly faces a pedestrian(s) who is passing across the road. The ADS selects a proper maneuver to avoid a vehiclepedestrian(s) collision. Now, if there is a barrier in the vehicle's new path and ADS has not detected it for some reason in advance, the active seat rotation technique will not work effectively. ADS will not have had enough time to change the orientation of the seats. Hence, it is necessary to investigate the safety of nonstandard seating configurations.

Human body models are another important element in crash simulation tests. The majority of researchers' interests have been the frontal and lateral impact's effect on the human's head, neck, chest, abdomen and pelvis. Most of these investigations were carried out on post-mortem human subjects (PMHS) [9, 15, $18,22,23]$. Because of difficulties in keeping the condition of PMHSs, they cannot be used for multiple tests. PMHSs are used in research to deal with the crash's effects on the human body. Dummies, as full-scale anthropomorphic test instruments, are used for doing tests and measurements [24]. They can simulate a body's dimensions, weight proportions and articulations, as well. Dummies are used in the automotive industry for regulations and doing tests based on consumers' requests. Serious damages that happen in consequence of crash tests, dramatically decrease the dummies' measurement's accuracy.

Digital Human Modelling (DHM) can solve the aforesaid obstacles for PMHSs and the Dummies [1]. For instance, articulated rigid body (ARB) models are suitable for fast computation because of their simple manipulation. However, current developments in the computation's hardware, make it possible to use even more complex models. The finite element (FE) based models are another group of human bode models which are complex because of their precise anatomic model. In consequence, more simulation time is needed for running a test. Regardless of the time issue, these models can simulate injuries to internal organs. 
Hybrid models are being developed for having efficient time consumption for simulation(s) as well as the capability of collecting as much injury data for each body part. The Virthuman body model [3] [11] is selected in this study for simulating bodies. Since we considered four bodies in a car's interior, this model could help to run simulations faster, due to the limitations of our hardware facilities.

This study faces some limitations due to the vehicle interior and body models. Simulation results for a highly automated vehicle model in frontal collision condition with four identical bodies were not validated against experimental data in non-standard seating configurations. We dealt with a simplified schematic interior but for further studies, we suggest using available vehicle dimensions. It keeps the topic open for further studies. Present work has focused on a lowspeed frontal crash tests. For having a better view about occupants' safety, different crash directions and initial crash speeds have to be tested. Moreover, the models' seating posture is an effective parameter on bodily injury in a crash. Henceforth, further study is needed for other seating positions, such as, when they lean back. Finally, there is the Virthuman model itself, which possesses the decent capability to present different ages and genders in crash scenarios. The authors recommend extending the study for a range of ages and genders, to collect a valuable database of crash simulations. As far as the Virthuman model is made on a Multi-Body-System basis, it evaluates body injuries regarding kinematic values and loads on some certain nodes/joints. It is important to highlight the point, that Virthuman does not model tissues, muscles, brain, etc. None of these bodily injuries are considered by the algorithm for the evaluation of injury indexes.

\section{Methods}

Four identical Virthuman models were located in a simplified interior of an autonomous vehicle. Three-point seat belts were the only elements which restrict bodily motion and their feet were not affixed to the interior's floor. The whole model - bodies and interior - was subjected to a Low-impact speed's acceleration pulse $(30 \mathrm{~km} / \mathrm{h})$ in visual performance solution (VPS), PAM-CRASH module. An algorithm is available for Virthuman extracting time-dependent kinematic or loads' curves for specified nodes to compute the injury index per body's parts.

\section{$2.1 \quad$ Virthuman}

Virthuman is a hybrid, Multi-Body-System (MBS) model for representing a human's body in the field of traffic safety to investigate a crash scenario's effect on bodies [19]. The body's skeleton of this model is created based on MBS in which parts are connected by nonlinear springs and dampers. The models' skins 
are the integration of super elements, and nonlinear translational joints connect them to the MBS structure. The model is applicable for use in simulating different gender, age, height and weight categories of the population [6]. A scaling algorithm generates physical dimensions (geometry), mass, inertia and joint properties, concerning age, gender, height and mass distribution [3]. A virtual human model, Figure 1, in this survey represents a 30-year-old male with $176 \mathrm{~cm}$ height and a weight of $78 \mathrm{~kg}\left(50^{\text {th }}\right.$ percentile male $)$.

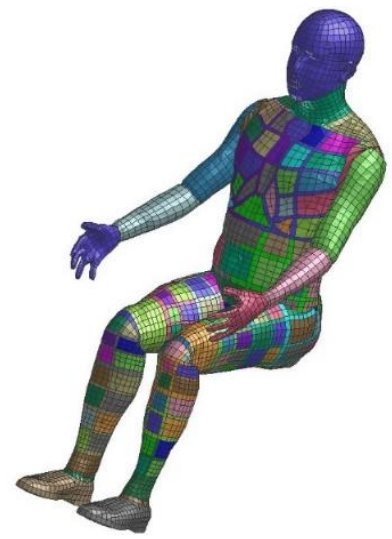

Figure 1

Virthuman model

\subsection{Vehicle Interior}

The simplified autonomous interior in the study contains upright walls, windows and seats. Its exterior dimension is $2646.09 \mathrm{~mm}$ length, $2070.7 \mathrm{~mm}$ width and $1420.67 \mathrm{~mm}$ height, Figure 2. The walls are covered by $50 \mathrm{~mm}$ Polyurethane foam (PUR). The geometry of the driver's seat of the Volvo xc7 Station Wagon is imported to VPS and has upright posture, Figure 2 [14]. The angle of the seat's cushion respect to the horizontal plane which is parallel to the floor of the vehicle's interior is 14.7 degree. Seat's back makes 20.35 degrees angle with an axis perpendicular to the vehicle's floor.

The seat is made from Polyurethane foam. This foam type is a highly versatile material and is widely used in the automobile industry. Foams act as useful shockabsorbers during vehicle motion and can dampen unwanted small vibrations that are caused by the road's roughness. The stress-strain curves for the Polyurethane foam used for the interior and seats in the present study are illustrated in Figure 3 [16]. 


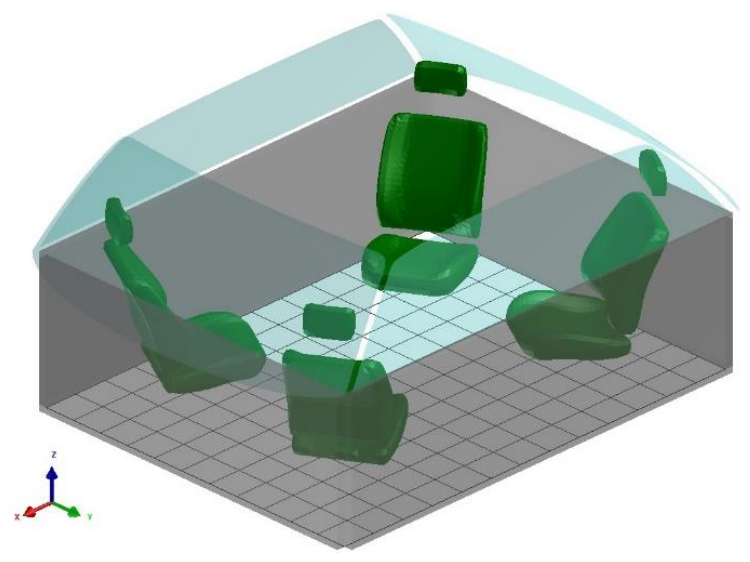

Figure 2

Polyurethane foam seats in the schematic interior

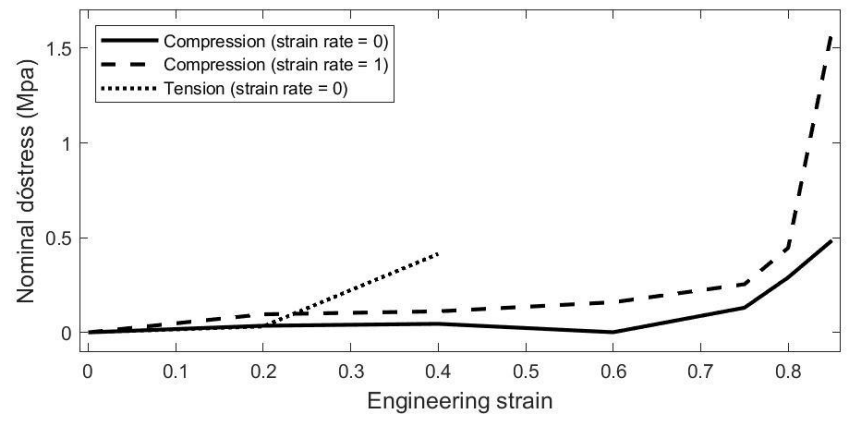

Figure 3

Polyurethane foam material properties

The three most desired seating configurations [8] are considered in this study, Figure 4. There is a common seating configuration where all passengers sit facing the front windshield. It provides two options for occupants, more specifically for people who are in the front row: lean their seats back and slightly move it horizontally to the front or rear for adjustment. We call it the standard seating configuration. First row seats in the standard seating configurations are rotated 180 degrees around an axis which is perpendicular to the interior floor plane and passes through the center of the seat's nodes. Meanwhile, rear row seats kept their orientations. We named this seating configuration, Face-to-Face. The final seating configuration was generated based on the Face-to-Face seating configuration, and we named it the Living Room. It looks like a family member sitting around a dining table. In the Living Room seating configuration, all passengers' seats in Face-to-Face configurations are rotated 30 degrees inward. 
In both standard and non-standard seating configurations, bodies are fastened to seats by three-point seat belts made from standard material for common passenger vehicles. Retractors are activated at the beginning of the simulation and push back belts to affix the bodies on the seats. The retractors' forces were set to $35 \mathrm{~N}$ [20]. No load limiter is assumed in this study. Shoulder and lap belt are made from double-layer fabric. This part was modelled in VPS by membrane element which has two perpendicular layers. For simplicity reasons, nonlinear beam elements are used for fixed belt's segments at retractor and anchor passing segments over Dring and buckle.
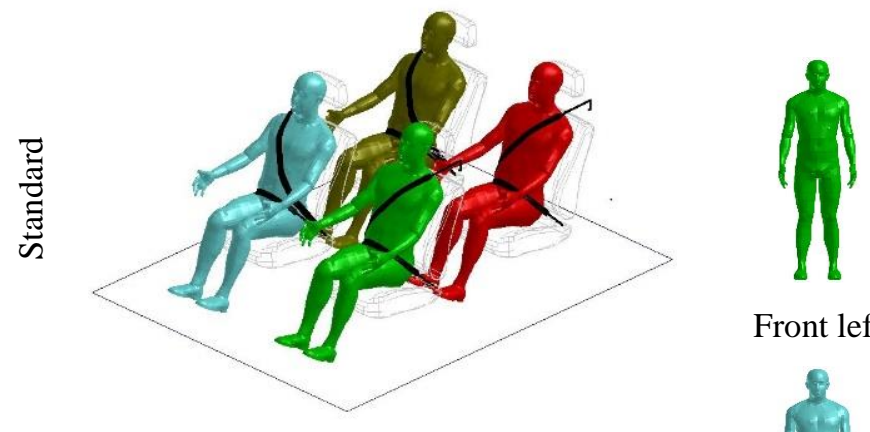

Front left
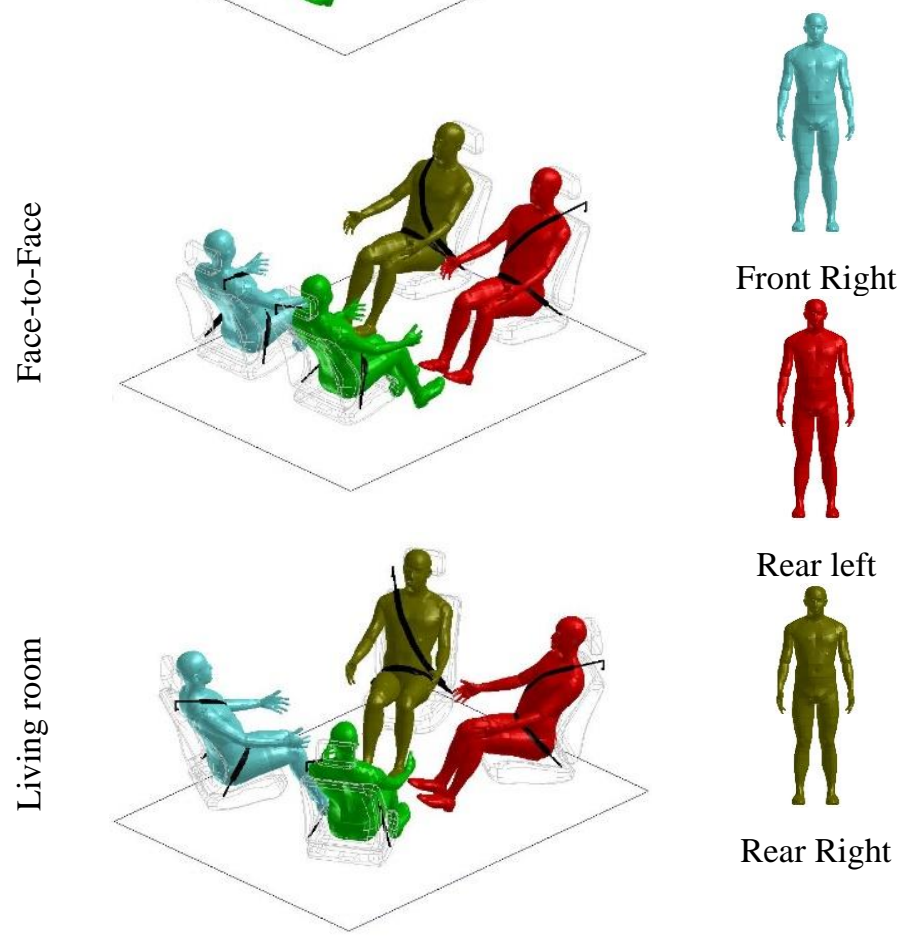

Rear Right

Figure 4

Seating postures 


\subsection{Part Injury Assessment}

An algorithm has been developed to assess the injury risk of the Virthuman model [2]. The program generates 779 time-dependent curves, such as, accelerations, forces and displacements of certain points and body parts. As an example of the acceleration's component and the absolute value of the head's center of gravity, is considered. The neck joints' bending moment plays an important role in computing injury index. The program measures the deflection of the Model's thorax segments during simulation to calculate its level of injury. Forces are key values for determining injuries to pelvis segments, Table 1. To determine injury indexes for breakable joints, the algorithm focuses on their forces and moments. For each model's part, the degree of injury is determined according to its relevant time-dependent curves have been mentioned already. The worst degree of injury in the time interval from the beginning to the current time step is assigned as the injury index for a particular segment. Therefore, the last animation step is read as a part of the injury's value. The injury values of models are age-dependent. For instance, the same load state causes worse injuries in children than in adults. The color evaluation according to the degree of injury is returned by four basic levels according to EuroNCAP consumer rating [4]. Either a small degree of injury or none is given by "Good". An injury level can also be "Acceptable" or "Marginal". However, fatal injuries are shown by the "Poor" level and cause a very serious degree of injury. For example, with the head injury index for HIC36<650, a "Good" level is assigned to the head. If HIC36>1000 the program returns "Poor". Injury indexes' limits are age-dependent but body's height, mass and gender are not considered for these ranges. The injury index metrics are available in the literature for a 6-year-old child, 20-year-old and 100-year-old adult, for each injury criteria $[5,10,12]$. For the other ages, the program uses linear interpolation to compute metrics.

Table 1

Injury criteria for Virthuman [20]

\begin{tabular}{|c|l|}
\hline Part & Injury criteria \\
\hline Head & HIC36, HIC15 \\
\hline Neck & Upper neck moment, tension and shear force \\
\hline Thorax & Deflection (front, side), Viscous criterion (front, side) \\
\hline Abdomen & Compression force \\
\hline Pelvis & Pubic peak force \\
\hline Femur & Bending moment, Compression force \\
\hline Knee & Moments \\
\hline Tibia & Bending moment, Compression force \\
\hline
\end{tabular}




\section{Simulation}

When a vehicle collides into a barrier, the vehicle comes to an immediate stop, while the occupants keep moving, according to Newton's laws of motion. Henceforth, the present study uses an equal case and supposes that the car's body is pushed back from the rest-position using a given acceleration, that corresponds to the initial car crash speed. The acceleration is applied to the geometry center of the model's nodes - human body models and interior - in the horizontal direction. The model has a single degree of freedom of movement in the direction of the crash. Three simulations are run in this study: Standard, Face-to-Face and Living Room seating configurations. A two-step simulation in VPS is planned for running the tests.

- To prevent any simulation error that may occur due to initial contact's penetration between bodies and seats, gravity acceleration is applied on body models, as well as the seats, for $50 \mathrm{~ms}$. Body models are released slightly above the seats from the rest-position, at the beginning of this step. In this time interval, the acceleration pulse equals to zero, Figure. 5, and the gravity is the only external acceleration field acting on models' nodes. Meanwhile, seat belts are pulled back by their retractors. By the end of this step, the seat belts are locked and the seats are deformed.

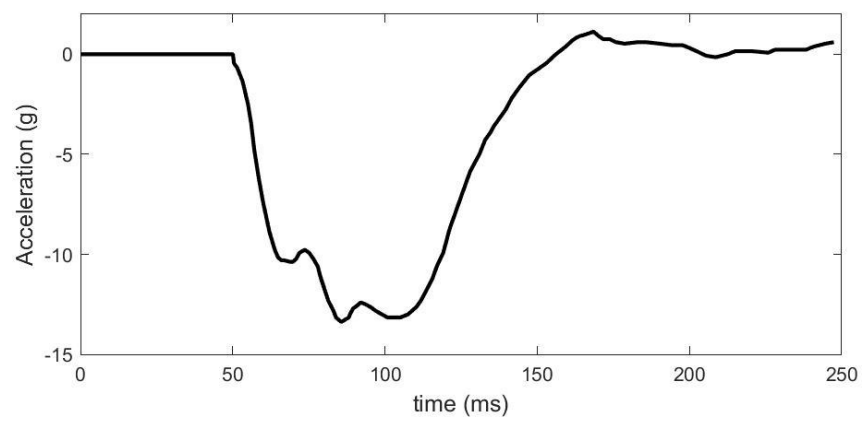

Figure 5

Prescribed vehicle's acceleration vs. time

The crash's acceleration pulse for $30 \mathrm{~km} / \mathrm{h}$ [17] is applied to the center of the model's nodes for $200 \mathrm{~ms}$. The models' motion data are recorded versus time for post-processing analysis. Occupants' kinematic responses, in standard and nonstandard seating configurations, are illustrated for selected times in Figure 6. 


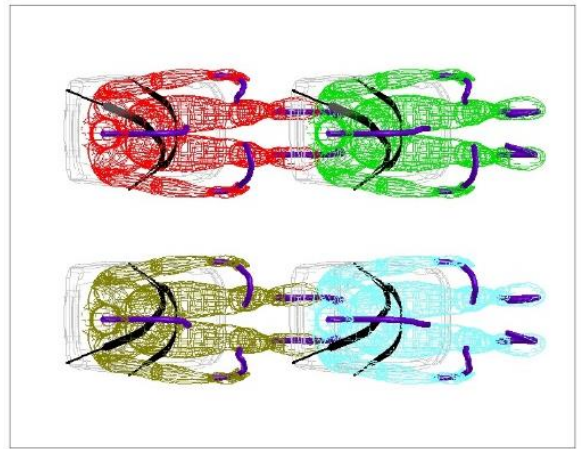

Standard seating configuration

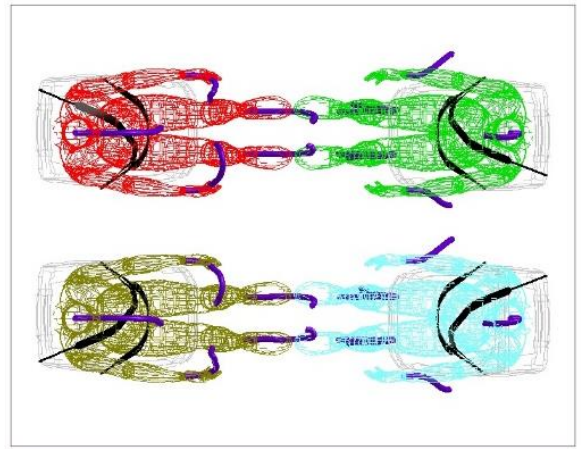

Face-to-Face seating configuration

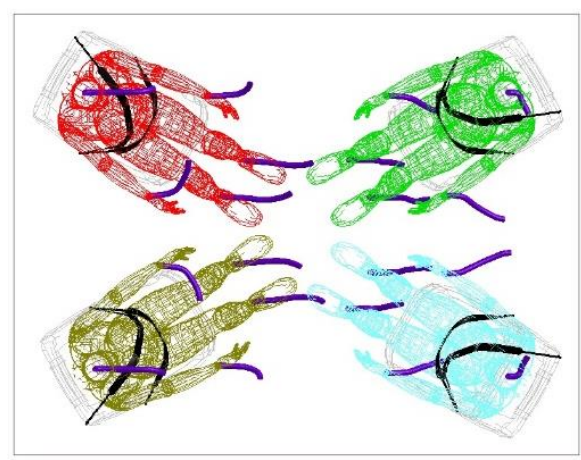

Living room seating configuration

Figure 6

Occupants' body parts' trajectory (head, wrists and feet) in a frontal collision in different seating configurations

\section{Post-Processing Results}

Occupants' injury index assessments are collected for the Standard seating position in Figure 7. Four bodies are identical and the seats' direction is the same as the crash orientation. One expects to have quite similar index injury for all occupants. Forces/moments in segments and joints of bodies' parts, these do not exceed metric limitations used by the algorithm [2, 5, 10, 12], as all bodily parts are in "Good" condition in a crash, except for their thoraxes and abdomens. During the crash, bodies are bent forward while the shoulder and lap belts, which are locked and do not have any motion, pull back the bodies. Chest's segments that are acting as rigid bodies and press translational joints which are connected to the models' MBS structure. The side's deflection of the thoracic segment which is 
in contact with shoulder belts exceeds $42 \mathrm{~mm}$, a defined injury criteria limitation for thoracic side's deflection. Hence, the thorax's injury index for all bodies is Poor".

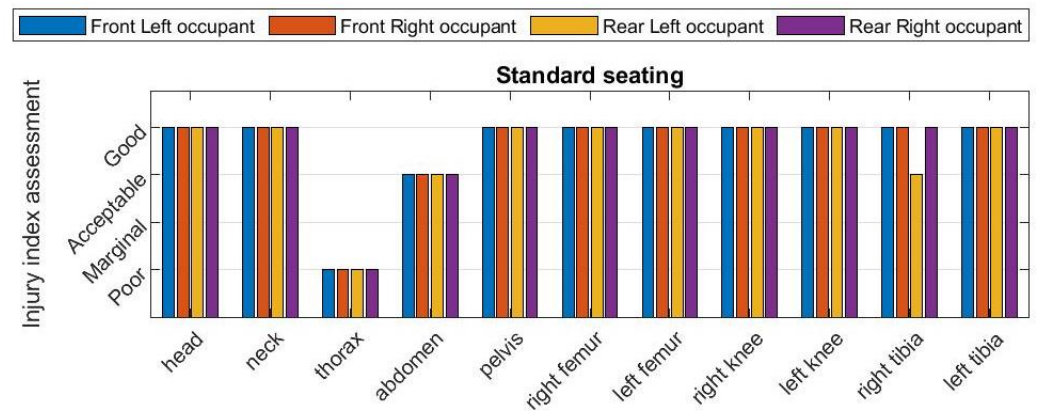

Figure 7

Standard seating configuration's Injury index assessment per occupants

There are minor changes in compression forces of the bodies' abdomen joints because this section of the body is supported by the seat's foam. The foam can be deformed easier and even more than the body' segment. It helps abdominal forces will not be more than $1.75 \mathrm{kN}$, a defined value for the abdomen's injury criteria. Therefore, one has an acceptable injury index for abdomens. A difference is seen for injury index of Rear Left occupant's right tibia because rigid bodies contact happened. Some nodes under seats' cushions were selected as rigid nodes. All seats were identical however bodies were located and fastened on their seats individually. In a step during simulation distance between Rear Left occupant's right foot and rigid nodes under Front Left's cushion was less than a value which was set for contacts. In this occasion, VPS solver interpreted it as a rigid-to-rigid contact that makes a high jump in contact forces. Subsequently, it generates a pick in compression force of the model's right tibia. Therefore, the injury assessment algorithm evaluated "Acceptable" level for this body part. Front row occupants' feet also hit to the front of the interior. But it was covered with Polyurethane foam and it was deformed to absorb energy. Hence, tibiae' index of injury for Front row occupants, remain in "Good" condition.

Figure 8, demonstrates injury indexes for occupants in the Face-to-Face seating configuration. In this configuration, the front row seats are rotated 180 degrees and the rear row seats' position remain unchanged. Therefore, it is expected that the front row occupants' injury indexes are changed because they somehow experience a crash from their backside. Major differences are seen in the front-row occupants' thoraxes and femurs. These bodies' reaction to the crash, pulse push the models' trunk to seats' back. The seat's foam absorbs a part of the crash energy. This motion causes bodies to receive minimal contact with the shoulder belt, which reduces the thoracic segments' deflection. In consequence, these parts' deflection values are lower than the metric limits defined in the algorithm, and their injury index is improved from "Poor" to "Acceptable". 


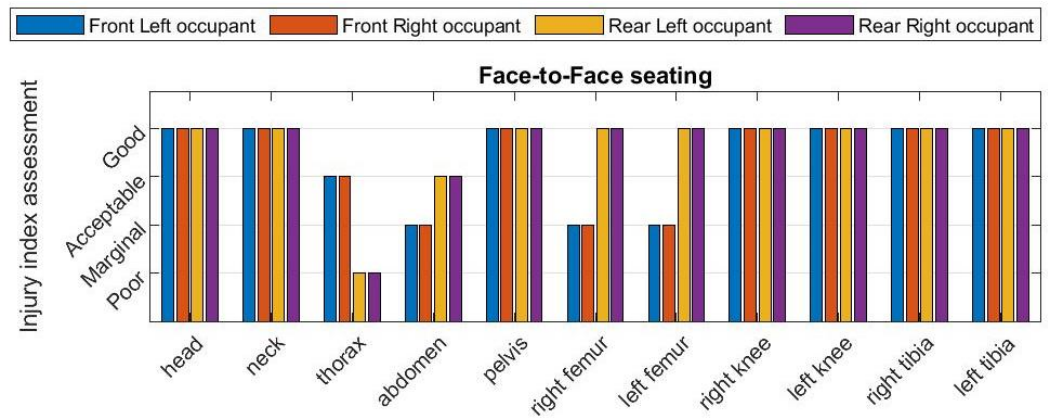

Figure 8

Face-to-Face seating configuration's Injury index assessment per occupants

Actually, at the end of simulation time when the trunk of front rows' occupants hit to the shoulder belt in a rebound, their injury index is changed to "Acceptable". However, when these bodies are bent back a little around their pelvis, compression forces on their abdomen are increased - slightly more than $1.75 \mathrm{kN}$ because bodies are pushed by lap belts. This means we have a change in injury index from "Acceptable" to "Marginal". Another important injury index change related to bodies' femur. Returns to occupants' kinematic are shown in Figure 6, in which one can see that the tibiae of bodies' in the front row are pushed to the seat's cushion. On the other hand, it has been mentioned already that these bodies' abdomen motion is restricted by the lap belts. These two parameters lead to having an upsurge in femur forces that pass the $6.5 \mathrm{kN}$ limitation of femur's compression force. As a result, their injury index is reduced from "Good" to "Marginal".

Indexes of injury for occupants in the Living Room seating configuration are illustrated in Figure 9. Positions of occupants' seats in this configuration are similar to Face-to-Face but the only difference is they are rotated by 30 degrees inward. This rotation causes passengers to experience an oblique crash. The Front Left and Right occupants were rotated counter-clockwise and clockwise around their pelvises respectively, Figure 6 . These rotation of frontal raw bodies prevents tibiae collision to the front of the seat cushion, such as we have seen for Face-toFace. Forces are decreased to less than $5.5 \mathrm{kN}$ for the right femurs helps to improve injury indexes to "Acceptable".

Because the models' head does not hit the interior during crash's simulation and HIC36 metric are not exceeded from limitations considered by the software (HIC36<650), heads' injury indexes are at a "Good" level. Regarding models' necks, upper neck's joints are taken into account for determining injury indexes. The algorithm computes the upper neck's bending moment, shear and tension force in the midsagittal plane. For occasions such as the present study if joints' moment, shear and tension forces are less than $42 \mathrm{Nm}, 1.9 \mathrm{kN}$ and $2.7 \mathrm{kN}$ respectively a "Good" injury index is assigned to models' necks. While simulation 
was run in Living Room seating configuration all body models were rotated inward and there were contacts between shoulder belts and the lowest part of necks. The program does not consider forces/moments out of the midsagittal plane for neck injury index assessment. So Living Room seating configuration one also has "Good" injury index.

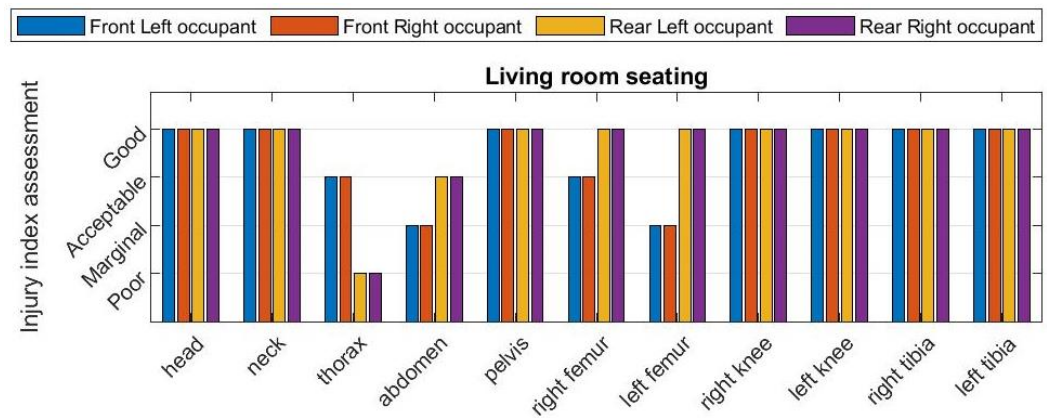

Figure 9

Living Room seating configuration's Injury index assessment per occupants

\section{Conclusions}

The present study investigated four identical $50^{\text {th }}$ percentile male Virthuman occupants' injury indexes, for three seating configurations, in a schematic interior of a highly autonomous vehicle. For frontal collision simulation, a low-impact speed's $(30 \mathrm{~km} / \mathrm{h})$ acceleration pulse was applied to the model. Subsequent injury indexes of body models' parts are investigated by the post-processing algorithm, which is available for the Virthuman model in the VPS Environment. Simulation results reveal bodies' thoraxes are the most vulnerable parts, in a frontal crash, in standard seating configurations, because of shoulder belts.

In non-standard seating configurations (Face-to-Face and Living Room), front row occupants' seats are rotated. Bodies sit face backwards so in a crash their back hit to the seats' back and the seats' foam absorbs the crash's energy. Because seat belts have been already locked in the first step of the simulation, contact between bodies and shoulder belts are reduced. As a consequence, the injury index in nonstandard seating configurations for front-row occupants' thoraxes are better than in standard seating configuration. However, the upper part of these bodies bend backwards and rise forces and moments on abdominal joints. The abdomen's injury index in non-standard configurations is worse than in standard configuration. Front-row occupants' femurs in crash hit the seats' cushion in the non-standard seating configuration. This means their injury index is worse than in the standard configuration. This issue can be solved by adding extra foam to the cushion, as a lower leg rest, for restricting leg motion. Another possible solution which can be tested, is to affix feet to the interior's floor, in a test. 
Returning to simulation results, based on the settings that were taken into account in the present study, in general, the Living Room seating configuration is safer in a low-speed frontal crash, over the Standard and Face-to-Face, in regards to a body injury index. However, it is worth stressing, that no internal organs, tissues or brain injuries were considered in the latter mentioned outcome due to the Virthuman limitations. Further studies and validation of results against experimental tests are recommended.

\section{Acknowledgement}

This work was supported by the European Regional Development Fund-Project "Application of Modern Technologies in Medicine and Industry" (No. CZ.02.1.01/0.0/0.0/17_048/0007280).

\section{References}

[1] DHM and Posturography, ed. S. Scataglini and G. Paul. 2019: Academic Press

[2] ESI, Virthuman Postprocessing Manual (VPS Explicit MBS Model, Model Version 1.1) Rev. 1, 2016

[3] ESI, Virthuman User's Manual (VPS Explicit MBS Model, Model Version 1.1) Rev. 1, 2016

[4] EuroNCAP, ASSESSMENT PROTOCOL - ADULT OCCUPANT PROTECTION, 6.0, 2013

[5] Forman, J. L., et al. Fracture tolerance related to skeletal development and aging throughout life: 3-point bending of human femurs. in IRCOBI Conference Proceedings, Dublin, Ireland. 2012

[6] Hyncik, L., et al., On scaling virtual human models, 2013

[7] Jin, X., et al. Occupant kinematics and biomechanics with rotatable seat in autonomous vehicle collision: a preliminary concept and strategy. in 2018 IRCOBI Conference Proceedings. IRCOBI. 2018

[8] Jorlöv, S., K. Bohman, and A. Larsson. Seating Positions and Activities in Highly Automated Cars-A Qualitative Study of Future Automated Driving Scenarios. in The International Conference on the Biomechanics of Impact (IRCOBI) 2017

[9] Kang, Y. S., et al. Head and Neck Responses of Post Mortem Human Subjects in Frontal, Oblique, Side and Twist Scenarios. in The International Conference on the Biomechanics of Impact (IRCOBI) 2018

[10] Kleinberger, M., et al., Development of improved injury criteria for the assessment of advanced automotive restraint systems. 1998, 4405(9): pp. $12-17$ 
[11] Kovar, L. and J. Hlucha, ESI VIRTHUMAN models for impact, in DHM and Posturography, S. Scataglini and G. Paul, Editors. 2019, Academic Press. pp. 169-185

[12] Mertz, H. J. and L. M. Patrick, Strength and response of the human neck. 1971: pp. 2903-2928

[13] Nie, B., et al., Seating preferences in highly automated vehicles and occupant safety awareness: A national survey of Chinese perceptions. Traffic injury prevention, 2020: pp. 1-7

[14] Ozmumcu, S. Volvo xc7 Station Wagon Seat. 2012; Available from: https://grabcad.com/library/automobile-seats

[15] Shaw, G., et al., Impact response of restrained PMHS in frontal sled tests: skeletal deformation patterns under seat belt loading. Stapp Car Crash Journal, 2009, 53: pp. 1-48

[16] Špirk, S., M. Křŕžek, and Š. Jeníček. Polyurethane foam behaviour during impact. in MATEC Web of Conferences. 2018, EDP Sciences.

[17] Vezin, P., et al., Comparison of Hybrid III, Thor- $\alpha$ and PMHS response in frontal sled tests, 2002

[18] Viano, D. C., et al., Biomechanics of the human chest, abdomen, and pelvis in lateral impact. Accident Analysis I\& Prevention, 1989, 21(6): pp. 553574

[19] Vychytil, J., et al., Scalable multi-purpose virtual human model for future safety assessment, 2014

[20] Vychytil, J. and S. Špirk, Numerical analysis of passenger kinematics and injury risks during a railway vehicle collision: The effect of safety belts. 2020, 14(1)

[21] $\mathrm{Wu}, \mathrm{H}$., et al., Occupant kinematics and biomechanics during frontal collision in autonomous vehicles-can rotatable seat provides additional protection? Computer Methods in Biomechanics and Biomedical Engineering, 2020: pp. 1-10

[22] Yoganandan, N., et al. Lateral neck injury assessments in side impact using post mortem human subject tests. in Annals of Advances in Automotive Medicine/Annual Scientific Conference. 2011, Association for the Advancement of Automotive Medicine

[23] Yoganandan, N., et al., Use of postmortem human subjects to describe injury responses and tolerances. Clinical anatomy, 2011, 24(3): pp. 282293

[24] Yoganandan, N. A., et al. Sled tests using the THOR-NT device and post mortem human surrogates in frontal impacts. in The International Conference on the Biomechanics of Impact (IRCOBI) 2012 